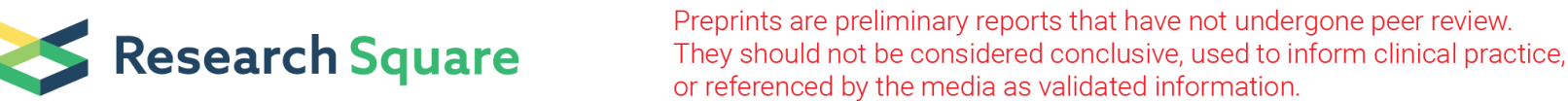

\section{Long noncoding RNA ARHGAP5-AS1 inhibits migration of breast cancer cell via stabilizes SMAD7 protein}

\section{Chen-Long Wang}

Xuzhou Medical University https://orcid.org/0000-0002-6965-2774

Jing-Chi Li

Shanghai Jiao Tong University School of Medicine

Ci-Xiang Zhou

Shanghai Jiao Tong University School of Medicine

Cheng-Ning Ma

Shanghai Jiao Tong University School of Medicine

\section{Di-Fei Wang}

Shanghai Jiao Tong University School of Medicine

\section{Lu-Lu Wo}

Shanghai Jiao Tong University School of Medicine

\section{Ming He}

Shanghai Jiao Tong University School of Medicine

\section{Guo-Qiang Chen}

Shanghai Jiao Tong University School of Medicine

Jian-Rong He

Shanghai Jiao Tong University Medical School Affiliated Ruijin Hospital

Qian Zhao ( $\nabla$ qzhao@shsmu.edu.cn )

https://orcid.org/0000-0001-8103-8251

\section{Primary research}

Keywords: ARHGAP5-AS1, breast cancer, metastasis, SMAD7

Posted Date: August 21st, 2020

DOl: https://doi.org/10.21203/rs.3.rs-43516/v2

License: (c) (i) This work is licensed under a Creative Commons Attribution 4.0 International License.

Read Full License 
Version of Record: A version of this preprint was published at Breast Cancer Research and Treatment on August 9th, 2021. See the published version at https://doi.org/10.1007/s10549-021-06286-5. 


\section{Abstract}

Background: Tumor metastasis is the main cause of death from breast cancer patients and cell migration plays a critical role in metastasis. Recent studies have shown long non-coding RNAs (IncRNAs) play an essential role in the initiation and progression of cancer. In the present study, the role of a LncRNA, ARHGAP5-AS1 in breast cancer was investigated.

Methods: Bioinformation was analyzed for the expression of ARHGAP5-AS1. qRT-PCR was conducted to verify the expression of ARHGAP5-AS1 in breast cancer specimens. Transwell migration assays and Factin staining were utilized to estimate cell migration ability. RNA pulldown assays and RNA immunoprecipitation were used to prove the interaction between ARHGAP5-AS1 and SMAD7. Western blot and immunofluorescence imaging were used to examine the protein levels. Dual luciferase reporter assays were performed to evaluate the activation of TGF- $\beta$ signaling.

Results: Compared to MDA-MB-231 cells, the expression of LncRNA ARHGAP5-AS1(NR_027263) was significantly suppressed in its highly metastatic subtype MDA-MB-231-LM2 cells. Functional study showed ARHGAP5-AS1 could inhibit cell migration via suppression of stress fibers in breast cancer cell lines. Afterwards, SMAD7 was further identified to interact with ARHGAP5-AS1 by its PY motif and thus its ubiquitination and degradation was blocked due to reduced interaction with E3 ligase SMURF1 and SMURF2. Moreover, ARHGAP5-AS1 could inhibit TGF- $\beta$ signaling pathway due to its inhibitory role on SMAD7.

Conclusions: Overall, these findings demonstrate that ARHGAP5-AS1 inhibits breast cancer cell migration and could server as a novel biomarker for breast cancer metastasis and a potent target for the treatment in the future.

\section{Background}

Breast cancer is one of the most common cancer and accounts for $29 \%(246,660)$ of all new cancers among the worldwide women [1]. According to the latest statistics, released by the American Cancer Society, about 276,480 new cases of invasive breast cancer are expected to be diagnosed in the United States in 2020, which means one in eight women will get breast cancer during their lifetime. In addition, 42,170 deaths per year makes breast cancer the second leading cause of cancer-related mortality among women in the USA [2]. The main cause of death from breast cancer is due to metastasis to other organs (e.g. bone, lung, brain and liver) [3]. Nevertheless, the mechanisms underlying the metastatic dissemination remain poorly understood, which causes a critical barrier for breast cancer therapy. Cell migration is regarded to be one of the essential step involved in metastasis [4]. Reduced migration suppressed the dissemination of cancer cells in a breast cancer mouse model [5]. It is well characterized that cancer cell could achieve migration via rearrangement of cytoskeleton [6]. Thus, expanding the understanding of mechanisms underlying cytoskeleton reorganization may promote the understanding of cell migration and hence cancer metastasis. 
Recent studies have shown that long non-coding RNAs (IncRNAs) play an essential role in the initiation and progression of cancer. LncRNAs are gene transcripts which have more than 200 nucleotides in length and no potential of translation [7]. LncRNAs have been proposed to carry out diverse functions, including transcriptional regulation in cis or trans, organization of nuclear domains, and regulation of proteins or RNA molecules [8]. It is now widely understood that IncRNAs could identify cellular pathologies such as cancer, provide prognostic value, or even serve as therapeutic options for cancer patients [9]. For example, over-expression of the IncRNA HOTAIR in early-stage, surgically resected breast cancer is highly predictive of progression to metastatic disease and overall survival [10]. Silencing the expression of the IncRNA GUARDIN triggered apoptosis and senescence, enhanced cytotoxicity of additional genotoxic stress and inhibited cancer xenograft growth. Thus, GUARDIN may constitute a target for cancer treatment [11].

The transforming growth factor b (TGFb) signaling pathway is a key player in metazoan biology, and its misregulation can result in tumor development. Pathological forms of TGFb signaling promote tumor growth and invasion, evasion of immune surveillance, as well as cancer cell dissemination and metastasis [12]. Smad6 and Smad7 are inhibitory Smads that negatively control TGF $\beta$ pathway activity in response to feedback loops and antagonistic signals [13]. SMAD7, as one of the key inhibitor of TGFb signaling, negatively regulates the whole pathway via multiple mechanisms [14]. In the cytoplasm, SMAD7 can compete the binding site of type-1 TGFb receptor (TGFb R1) with SMAD2/3 and hence inhibit signal transduction by blocking phosphorylation of SMAD2/3 [15]. Besides, SMURF1 and SMURF2 are recruited to TGFb R1 by SMAD7 and induce the degradation of receptors [16]. Meanwhile in the nucleus, SMAD7 disrupts the binding of SMAD2/3/4 complex with DNA[17].

In our present study, we identified a novel IncRNA, Rho GTPase activating protein 5 antisense RNA 1 (ARHGAP5-AS1), which expression is downregulated in highly metastatic breast cancer cell line MDA-MB231-LM2. Functional study showed that ARHGAP5-AS1 could inhibit migration of breast cancer cells through the inhibition of stress fibers. Moreover, we found that ARHGAP5-AS1 could interact with SMAD7 and stabilized SMAD7 protein via blocking the interation between SMAD7 and its E3 ligase (SMURF1 \& SMURF2), and hence inhibited its ubiquitination and degradation. Further more, ARHGAP5-AS1 could inhibit TGF $\beta$ signaling pathway by upregulation of TGFb R1. Taken together, these findings demonstrate that ARHGAP5-AS1 could server as a novel biomarker for breast cancer metastasis and a potent target for the treatment in the future.

\section{Materials And Methods}

\section{Cell lines and cell culture}

Breast cancer cell line MDA-MB-231-LM2 was obtained from Guohong Hu (Institute of Health Science, Chinese Academy of Sciences, Shanghai, China). MDA-MB-231 was provided by Ming-Yao Liu (East China Normal University, Shanghai, China). SKBR3 and BT549 were purchased from the cell bank of the Chinese Academy of Science (Shanghai, China). MDA-MB-231-LM2 was cultured in DMEM medium (Hyclone) supplemented with 10\% FBS (Gbico). MDA-MB-231 was cultured in Leibovitz L-15 medium 
(Gibco) supplemented with 10\% FBS. SKBR3 and BT549 were cultured in RPMI 1640 (Hyclone) medium supplemented with $10 \% \mathrm{FBS}$. All cell lines were incubated at $37{ }^{\circ} \mathrm{C}$ in a humidified atmosphere of $5 \% \mathrm{CO}_{2}$ and $95 \%$ air except for MDA-MB-231 which were raised in a humidified atmosphere containing $100 \%$ air.

\section{RNA extraction and quantitative real-time PCR}

Total RNA of cancer tissues and cell line were extracted from Trizol reagents (Invitrogen\#15596018) according to the manufacturer's instructions. CDNA was obtained by AMV reverse transcription system (TAKARA\#2621) followed the manufacturer's protocol. Quantitative real-time PCR was performed with SYBR Green PCR master mix reagent (ABI\#4472908) and specific primers for SMAD7-F/R (ATGCTGTGCCTTCCTCCGCTG/CCACGCACCAGTGTGACCGA) and ARHGAP5-AS1-F/R (GGCCCCTGATTCAGTACGTT/GCGTGAACAGGGGTCTTTTG). Data analysis of ARHGAP5-AS1 expression in breast cancer cell lines was normalized by internal control 28S RNA and evaluated using $2^{\Delta \Delta C_{t}}$ method, while data analysis of breast cancer tissues was normalized by $18 \mathrm{~S}$ RNA and presented by $-\Delta \mathrm{Ct}$.

\section{LncRNA in vitro Translation}

This experiment was performed according to the manufacturer's instructions (Promega\#L1170). Briefly, 1 $\mu \mathrm{g}$ of circular plasmid pcDNA3.1(+)-ARHGAP5-AS1 and pcDNA3.1(+)-ARHGAP5-AS1-AS were used in the translation reaction. The tubes were incubated $1 \mathrm{~h}$ at $30^{\circ} \mathrm{C}$. Then, $2 \mu \mathrm{L}$ of reaction liquid was added into $15 \mu \mathrm{L}$ SDS Loading Buffer and boiled 3 times at $105^{\circ} \mathrm{C}$ on incubator. Streptavidin-HRP (CST\#3999) was utilized to detect products of translation.

\section{Immunofluroscence staining}

F-actin staining was performed according to the manufacturer's instructions (Invitrogen\#R415). Briefly, $1.5 \times 10^{5}$ cells transfected with si-NC/si-ARHGAP5-AS1 or pcDNA3.1(+)-VEC/pcDNA3.1(+)ARHGAP50AS1 for $48 \mathrm{~h}$ were seeded onto coverslips in 24-well culture plate. A total volume of $200 \mu \mathrm{L}$ containing $4 \mu \mathrm{L}$ Rhodamine Phalloidin was used per well and incubated $30 \mathrm{~min}$ at room temperature. As for SMAD7 staining, the FITC conjugated antibody (Santa Cruz\#sc-365846 FITC) was used at the dilution rate of $1: 50$ in 1\% BSA and incubated overnight. Immunofluorescence pictures were taken by Nikon A1R confocal microscope (Nikon, Kanagawa, Japan).

\section{Transwell migration assay}

Cell migration ability was determined by transwell chambers (Corning\#3422). The experimental procedure was described elsewhere [18]. Briefly, $2 \times 10^{4}$ cells transfected with si-NC/si-ARHGAP5-AS1 or pcDNA3.1(+)-VEC/pcDNA3.1(+)-ARHGAP50AS1 for $48 \mathrm{~h}$ were seeded into transwell chambers. $18 \mathrm{~h}$ later, migrated cells were staining by crystal violet. Pictures were taken by Nikon Eclipse Ti microscope (Nikon, Kanagawa, Japan).

\section{RNA Fluorescent in situ hybridization}


This experiment was performed with Fluorescent In Situ Hybridization Kit (Ribo\#R11060.1) according to the manufacturer's protocol. Briefly, $1.5 \times 10^{5}$ MDA-MB-231 cells or LM2 cells was seeded onto cover slides in 24-well culture plate. LncRNA ARHGAP5-AS1 was detected by specific probe with Cy3 labeling. Fluorescence pictures were taken by Nikon Eclipse Ti microscope (Nikon, Kanagawa, Japan).

\section{Protein microarray screening}

Sense and antisense IncRNA were transcripted in vitro with Cy 5 labeling. Synthesized IncRNA were incubated with HuProt ${ }^{\mathrm{TM}}$ microarray (CDI Laboratories, Inc.) which contained more than 20000 full length human proteins with GST tag. This procedure was completed by Wayen biotechnologies (Shanghai), Inc. according to the manufacturer's instructions. In brief, proteome microarrays were blocked with blocking buffer for $1 \mathrm{~h}$ at room temperature before incubation of transcripted IncRNA in blocking buffer with microarray at room temperature for $1 \mathrm{~h}$. TBST followed by MilliQ water were used to wash the microarrays three times for $5 \mathrm{~min}$. Dried slides were scanned using GenePix 4000B. Images were analyzed using GenePix Pro 6.0.

\section{RNA pull down assays}

In vitro transcription of antisense or sense ARHGAP5-AS1 was achieved by T7 RNA Polymerase (Roche\#10881767001) according to the manufacturer's instructions. Meanwhile, $2 \mu \mathrm{L}$ of Biotin RNA Labeling Mix (Roche\#11685597910) was added into the transcription reaction. Cell lysates were prepared by sonication in BufferA [ $150 \mathrm{mM} \mathrm{KCl}, 25 \mathrm{mM}$ Tris pH7.4, $5 \mathrm{mM}$ EDTA, $0.5 \mathrm{mM}$ DTT, 0.5\% NP40 supplemented with $1 \mathrm{mM}$ PMSF (Bytotime\#ST506) and Cocktail (Millipore\#539134)]. $15 \mu \mathrm{g}$ transcripted RNA was added into lysates of $1 \times 10^{7}$ cells and incubated at $4{ }^{\circ} \mathrm{C}$ for $2 \mathrm{~h}$ in a rotary shaker. Streptavidin Magnetic Beads (NEB\#S1420) was utilized to enrich RNA-protein complex before washing five times in BufferA and elution in SDS Loading buffer. Eluted proteins were separated by SDS-PAGE for western blot.

\section{RNA Immunoprecipitation (RIP) assays}

RIP assays were performed by utilizing the Millipore Magna RIP Kit (\#17-700) according to the manufacturer's protocol. Bound RNA was extracted by Trizol reagents and subjected to real-time PCR using specific primers.

\section{Dual luciferase reporter assays}

$4 \times$ SBE was synthesized and cloned into pGL4.27 vector. 293T cells were cultured in 24-well tissue culture plates $24 \mathrm{~h}$ before transfection. Each three wells were transfected with pGL4.27-4×SBE/EV, pcDNA3.1(+)ARHGAP5-AS1/EV and Renilla. $5 \mathrm{ng} / \mathrm{mL}$ TGF 31 was added into each well $48 \mathrm{~h}$ after transfection. After 6 h stimulation, 293T cells was lysed by Passive Lysis Buffer. Luciferase activity were detected according to manufacturer's protocol (Promega\#E1910).

\section{Western blot}


This experimental procedure was described elsewhere [18]. Antibodies used in this article: SMAD7 (Sigma-Aldrich\#SAB1404041-100UG), $\beta$-actin (MBL\#PM053-7), LARP1 (ABCAM\#AB86359), GAPDH (Santa Cruz\#SC-32233), FLAG (Sigma-Aldrich\#F1804), SMAD7-B8-FITC (Santa Cruz\#SC365846FITC), pSMAD-2/3 (CST\#9510), SAMD2/3 (CST\#8685), TGF-3R1(Sigma-Aldrich\#SAB4502958-100UG), LaminB (Santa Cruz\#SC6216), HIS (Beyotime\#AH367).

\section{Statistical analyses}

All experiments were repeated at least three times or otherwise mentioned. The p-values for comparison between two groups were obtained by Student's t-test (two-tailed). All values were presented as Mean \pm STD and the p-value $\otimes 0.05$ was considered to be statistically significant.

\section{Results}

\section{ARHGAP5-AS1 is a downregulated LncRNA in agressive breast cancer cells}

To investigate the downregulated LncRNAs involved in breast cancer metastasis, MDA-MB-231-LM2 which is a more aggressive subtype of MDA-MB-231 was utilized [19]. LncRNA expression profiling of MDA-MB-231-LM2 and MDA-MB-231 cells was explored by RNA sequencing (Figure 1A). 80 RNA transcripts were downregulated in MDA-MB-231-LM2 cells (fold change $₫ 2$ ). Based on the identity, length and suitable regions for siRNA design, six downregulated IncRNAs were validated by qRT-PCR (Figure 1B). Three IncRNA genes (NR_027263, NR_030717, NR_046268) expressed lower ( $₫ 50 \%)$ and subjected to functional validation. For each LncRNA candidates, three siRNA fragments were designed and transfected into MDA-MB-231 cells. In order to verify the role of these LncRNAs on metastasis, transwell migration assays were performed. Suppression of NR_027263 showed highest promotion on cell migration among three candidates (Figure 1C). According to the location of NR_027263, it was named by Rho GTPase activating protein 5 antisense RNA 1 (ARHGAP5-AS1). To determine the identity of ARHGAP5-AS1, the translational potential of ARHGAP5-AS1 was detected by in vitro translational system. The results showed ARHGAP5-AS1 cannot encode any protein (Figure 1D), thus it's a long non-coding RNA. Taken together, the downregulation of ARHGAP5-AS1 promoted cell migration, suggesting a tumorsuppressive role of ARHGAP5-AS1 in breast cancer cells.

\section{ARHGAP5-AS1 inhibits migration of breast cancer cells}

According to previous results, downregulation of ARHGAP5-AS1 promoted cell migration of MDA-MB-231 cells. In order to verify the effects on other breast cancer cell line, SKBR3 and BT549 were utilized. The data showed that knockdown of ARHGAP5-AS1 promoted migration of MDA-MB-231 cells (Figure 2A) and SKBR3 cells (Figure 2B). On the contrast, over-expression of ARHGAP5-AS1 inhibited migration of LM2 cells (Figure 2C) and BT549 cells (Figure 2D). Interestingly, the observation of migrated cells showed enlarged lamellopodia in the knockdown group of MDA-MB-231 cells. This observation suggested the reorganization of cytoskeleton. Thus, we detected the distribution of the main component of cytoskeleton - F-actin by immunofluroscence. The results showed that knockdown of ARHGAP5-AS1 expression 
increased the formation of stress fibers in MDA-MB-231 cells (Figure 2E) as well as SKBR3 cells (Figure 2F). Conversely, over-expression of ARHGAP5-AS1 reduce the formation of stress fibers in LM2 cells (Figure 2G) as well as BT549 cells (Figure 2H). Taken together, ARHGAP5-AS1 inhibited migration of breast cancer cells through the inhibition of stress fibers formation.

\section{ARHGAP5-AS1 interacts with the PY motif of SMAD7}

LncRNAs can function as protein scaffold or microRNA sponge to regulate cellular processes in cytoplasm, while regulating gene transcription in cis or trans in the nucleus [9]. ARHGAP5-AS1 mainly existed in cytoplasm rather than in nucleus (Figure 3A), so we focused on the proteins interacting with ARHGAP5-AS1. In order to explore the mechanism of ARHGAP5-AS1 inhibiting migration of breast cancer cells, protein microarray screening was utilized to find the interacting protein with ARHGAP5-AS1. Firstly, sense and antisense ARHGAP5-AS1 was transcripted in vitro with Cy5 labeling (Figure S1A). The screening showed that there were 26 proteins interacting with ARHGAP5-AS1 sense excluding with antisense (Figure S1B). There were five proteins correlated to breast cancer progression among the interacting proteins with ARHGAP5-AS1 (Figure S1C). SMAD7 was proved to interact with ARHGAP5-AS1 by RNA pull down assay (Figure 3B). More importantly, the enrichment of SMAD7 and ARHGAP5-AS1 complex could be detected by RNA immunoprecipitation (Figure 3C). Furthermore, truncations with Flag tag were constructed to determine which domain of SMAD7 interacting with ARHGAP5-AS1. Precisely, truncation 1 lost $\mathrm{C}$-terminal $\mathrm{MH} 2$ domain while truncation 2 remained $\mathrm{N}$-terminal $\mathrm{MH} 1$ domain only, compared to full-length SMAD7 (Figure 3D). The interactions of different truncations with ARHGAP5-AS1 were detected by RNA pull down assay. The results showed that both full-length and truncation 1 SMAD7 were able to interact with ARHGAP5-AS1, but truncation 2 lost the interaction with ARHGAP5-AS1 (Figure 3E). It suggested that PY motif was essential for the interaction between ARHGAP5-AS1 and SMAD7. Taken together, ARHGAP5-AS1 interacted with SMAD7 through its PY motif.

\section{ARHGAP5-AS1 stabilizes SMAD7 via inhibition of ubiquitination of SMAD7}

PY motif of SMAD7 is responsible for the interaction between SMAD7 and its E3 ligase thus is important for the degradation of SMAD7 [20]. Due to the interaction of ARHGAP5-AS1 with PY motif of SMAD7, whether the stability of SMAD7 was influenced by ARHGAP5-AS1 was investigated. As expected, knockdown of ARHGAP5-AS1 reduced the protein level of SMAD7 in MDA-MB-231 cells and SKBR3 cells, while over-expression of ARHGAP5-AS1 increased the protein level of SMAD7 in LM2 cells and BT549 cells (Figure 4A-4B). The degradation of SMAD7 was controlled by ubiquitin and proteosome [21]. The proteosome inhibitor MG132 was applied to block the effects of ARHGAP5-AS1 on SMAD7. The results showed that MG132 blocked the degradation of SMAD7 caused by knockdown of ARHGAP5-AS1 (Figure 4C). In order to certify that the reduction of SMAD7 by knockdown of ARHGAP5-AS1 is due to the increase in degradation rather than the decrease in its mRNA level, the relative mRNA level of SMAD7 was detected by qRT-PCR. The results showed that whether knockdown or over-expression of ARHGAP5-AS1 had no influence on the mRNA level of SMAD7 (Figure S2A-S2B). Meanwhile, knockdown of ARHGAP5AS1 accelerated the degradation of SMAD7 when the protein synthesis inhibitor cycloheximide (CHX) 
stimulation applied (Figure 4D). Degradation of proteins in proteosome system results from polyubiquitination of proteins, so that the ubiquitination of SMAD7 was detected by immunoprecipitation. The results showed that over-expression of ARHGAP5-AS1 reduced ubiquitination of SMAD7 in 293T cells (Figure 4E). Moreover, ARHGAP5-AS1 had a negative effect on the interaction between SMAD7 and its E3 ligases SMURF1 and SMURF2 (Figure 4F). On the contrast, ARHGAP5-AS1 had no effect on the interaction between SMAD7 and its deubiquitinase OTUD1 (Figure S2C). Taken together, ARHGAP5-AS1 stabilized SMAD7 via reduction of ubiquitination as well as the degradation of SMAD7.

\section{SMAD7 mediates the negative effect of ARHGAP5-AS1 on cell migration}

As SMAD7 antagonizes TGF $\beta$ signaling, it has critical role in TGF- $\beta$ induced cytoskeleton rearrangement [22]. In order to determine whether SMAD7 mediated the suppression of cell migration by ARHGAP5-AS1, antisense DNA oligos was utilized to knockdown SMAD7 which mimicked knockdown of ARHGAP5-AS1. The knockdown efficiency was detected by western blot, and the results showed efficient knockdown of SMAD7 (Figure 5A). Immunofluorescence by TRITC-phalloidin showed increase in stress fiber formation in MDA-MB-231 cells with knockdown of SMAD7 (Figure 5B). As a result, knockdown of SMAD7 enhanced migration of MDA-MB-231 cells (Figure 5C). In sum, knockdown of SMAD7 had the similar effect on cell migration to knockdown of ARHGAP5-AS1. To investigate whether SMAD7 was able to rescue the promotion of migration by reduction of ARHGAP5-AS1, the stable over-expression of SMAD7 MDA-MB-231 cells was constructed. The expression of SMAD7 was detected by western blot. The results showed that knockdown of ARHGAP5-AS1 reduced the protein level of SMAD7 in plvx-EV cells, while ectopic expression of SMAD7 blocked the reduction of SMAD7 in plvx-SMAD7-OE cells (Figure 5D). Immunofluroscence of F-actin showed that knockdown of ARHGAP5-AS1 promoted the formation of stress fiber in plvx-EV cells, but had no effects in plvx-SMAD7-OE cells (Figure 5E). The similar results were achieved in migration assay (Figure 5F). Taken together, knockdown of SMAD7 exerted similar function to ARHGAP5-AS1. Moreover, ectopic expression of SMAD7 blocked the enhancement of migration by reduction of ARHGAP5-AS1 in MDA-MB-231 cells.

\section{Knockdown of ARHGAP5-AS1 promotes TGF- $\beta$ signaling via SMAD7}

Due to the different inhibitory mechanisms of SMAD7 in nucleus and cytoplasm [14], the subcellular location of SMAD7 was detected with knockdown of ARHGAP5-AS1. The results of immunofluorescence showed no change of SMAD7 location by knockdown of ARHGAP5-AS1 in MDA-MB-231 cells (Figure 6A). Besides, extraction of nuclear and cytoplasmic SMAD7 was consistent with observation in immunofluorescence (Figure 6B). Notably, expression of SMAD7 in cytoplasm was reduced by knockdown of ARHGAP5-AS1, which was similar to previous results (Figure 4A). SMAD7 turned off TGF $\beta$ signaling by inducing the degradation of TGFb R1[16]. In order to investigate the effects of ARHGAP5AS1 on TGF $\beta$ signaling, the components of TGF $\beta$ pathway were detected by western blot. The results showed increased expression of TGFb R1 and prolonged activation of TGF $\beta$ signaling, which in turn presented by increased phosphorylation of SMAD2 in response to TGF $\beta 1$ stimulation (Figure 6C). The similar pattern was showed by knockdown of SMAD7 (Figure 6D). Moreover, we constructed four SMAD- 
binding elements (SBE) into pGL4.27 vector and performed dual luciferase reporter assays in 293T cells. The results showed that the activity of firefly luciferase was enhanced by the treatment of TGF- $\beta$ and over-expression of ARHGAP5-AS1 inhibited the enhancement of luciferase activity (Figure 6E). Taken together, knockdown of ARHGAP5-AS1 promoted TGF $\beta$ signaling by upregulation of TGFb R1.

\section{Discussion}

This study provides the first evidence that LncRNA ARHGAP5-AS1 was downregulated in human breast cancer specimens. Furthermore, ARHGAP5-AS1 could inhibit cell migration via suppression of stress fibers in breast cancer cells. More importantly, to our knowledge it is the first IncRNA could interact with SMAD7, which is an important inhibitory Smads that negatively control TGFb signaling pathway. The present study demonstrated that ARHGAP5-AS1 impaired the degradation of SMAD7 via interaction with its PY motif, blocking the interation with its E3 ligase (SMURF1 \& SMURF2) and thus decreased its ubiquitination. Knockdown of SMAD7 mimicked the promotion of cell migration by knockdown of ARHGAP5-AS1. Meanwhile, ectopic expression of SMAD7 blocked the increase in migration and stress fiber formation by knockdown of ARHGAP5-AS1. Moreover, ARHGAP5-AS1 inhibited the formation of stress fiber and hence migration via suppression of TGF $\beta$ signaling by stabilized SMAD7.

ARHGAP5-AS1 located on the human chromosome 14 and was first identified as a transcriptional isoform of ARHGAP5 in gastric cancer by large-scale sequencing [23]. Non-coding RNA was considered as transcription "noise" in the past. However, more and more studies showed an emerging and diverse role in cancer pathways [9]. For example, IncRNA NKILA suppresses breast cancer metastasis by interaction with p65 and blocking IKB phosphorylation [24]. Here we illustrated that ARHGAP5-AS1 was a long non-coding RNA and its expression was downregulated in breast cancer tissues, which data were achieved from TCGA database. Meanwhile, the downregulation of ARHGAP5-AS1 was further evidenced in the collected human breast cancer tissues $(n=22)$, compared to the corresponding adjacent normal tissues, suggesting a tumor-suppressor role in breast caner. Thus, we investigated the role of ARHGAP5AS1 in initiation and progression of breast cancer. As a result, ARHGAP5-AS1 inhibited migration of breast cancer cells. Moreover, stress fiber formation was reduced by ARHGAP5-AS1. It's a pity that we have not studied the regulatory mechanism of ARHGAP5-AS1 expression in breast cancer cells. We thought there could be a possibility that the expression of ARHGAP5-AS1 is regulated by some transcription factors which expression are quite different in breast cancer tissues.

LncRNA functions as protein scaffold, which are already well established [25]. We demonstrated that ARHGAP5-AS1 interacted with PY-motif of SMAD7. PY motif is responsible for the interaction of SMAD7 with WW domain of E3 ligase [20]. Indeed, ARHGAP5-AS1 occupied PY motif and blocked the ubiquitination of SMAD7 by E3 ligase (SMRUF1 \& SMURF2), so that reduced the degradation of SMAD7 protein. SMAD7 is a powerful inhibitor of TGF $\beta$ signaling, antagonizing TGF $\beta$ signaling via multiple mechanisms [14]. TGF $\beta$ signaling pathway exerts tumor-suppressive effects in cancer initiation, yet it promotes epithelial to mesenchymal transition (EMT), cell invasion as well as migration in cancer progression $[26,27]$. The dual role of TGFb in cancer has long been noted, but its mechanistic basis, 
operating logic, and clinical relevance have remained elusive. TGF $\beta$ induces cytoskeleton reorganization by upregulation of RhoGEFs, such as NET1 [28] and GEFH1 [29]. While SMAD7 inhibits TGF $\beta$ induced actin reorganization and RhoA activation [22]. It is expectable that TGF $\beta$ induced stress fibers and cell migration could be blocked by ARHGAP5-AS1 due to its interaction with SMAD7 and increased the stability of the latter. However, the function of ARHGAP5-AS1 in breast cancer cells was detected without exogenous TGF $\beta$ stimulation. Tumor cells themselves are able to produce TGF- $\beta$ ligands [30],[31], which might be a reasonable explanation. Indeed, knockdown of SMAD7 mimicked the promotion effect of cell migration by knockdown of ARHGAP5-AS1. Furthermore, ectopic expression of SMAD7 was able to block the promotion of cell migration by knockdown of ARHGAP5-AS1. Above results were also achieved without TGF $\beta$ stimulation. To verify the effects of ARHGAP5-AS1 on TGF $\beta$ stimulated signaling, we further knockdown the expression of ARHGAP5-AS1 in MDA-MB-231 cells and these cells were starved for $12 \mathrm{~h}$ and treated with $0.3 \mathrm{ng} / \mathrm{mL}$ TGF $\beta 1$. Prolonged activation of TGF $\beta$ signaling was demonstrated by upregulation of TGFb R1 and increased phosphorylation of SMAD2 compared to the control cells. Besides, four fragments of SMAD-binding element (SBE) were cloned into pGL4.27 vector. ARHGAP5-AS1 overexpression showed reduced luciferase activity with stimulation of TGF $\beta$, proving the inhibitory role on TGF $\beta$ signaling.

In this study, we give a clear interpretation that ARHGAP5-AS1, which is downregulated in breast cancer tissue, suppresses the cell migration and stress fiber formation in breast cancer cells. Furthermore, SMAD7, which is an important inhibitory Smads that negatively control TGFb signaling pathway, is demonstrated to be interacted with ARHGAP5-AS1 and its protein level is regulated by ARHGAP5-AS1. Thus, our findings provide valuable clues toward understanding the mechanisms of human breast cancer progression and present an opportunity to develop more effective clinical therapies in the future.

\section{Conclusions}

Our experiments, results, and analysis indicated that ARHGAP5-AS1 inhibits cell migration through its inhibitory role on SMAD7 in breast cancer. ARHGAP5-AS1 could server as a novel biomarker for breast cancer metastasis and a potent target for the treatment in the future.

\section{Declarations}

\section{Authors' contributions}

Chen-Long Wang and Jing-Chi Li performed the molecular and cellular expriments. Ci-Xiang Zhou, Ming He and Guo-Qiang Chen contributed to experimental design. Cheng-Ning Ma performed RIP assay. Di-Fei Wang and Lu-Lu Wo performed in vitro translation assay. Jian-Rong He and Qian Zhao focused on writing the manuscript.

\section{Availability of data and materials}


The datasets during and/or analyzed the current study available from the corresponding author on reasonable request.

\section{Acknowledgements}

None.

\section{Consent for publication}

Not applicable.

\section{Competing interests}

The authors declare that they have no competing interests.

\section{Ethics approval and consent to participate}

The present study was performed after the approval of the Ethics Board of the Shanghai Jiaotong University, and all participants agreed to join the present study and signed written informed consent.

\section{Funding}

This work was supported by National Natural Science Foundation of China $(81472463,91213304$, $81470841,81772831)$ and its innovative group support (No.81721004).

\section{Abbreviations}

LncRNA: long non-coding RNA; ARHGAP5-AS1: Rho GTPase activating protein 5 antisense RNA 1; TGFb: transforming growth factor beta; SBE: SMAD binding elements; SMURF1 \& 2: SMAD specific E3 ubiquitin protein ligase 1 \& 2; OTUD1: OTU deubiquitinase 1.

\section{References}

1. Siegel RL, Miller KD, Jemal A: Cancer statistics, 2016. CA Cancer J Clin 2016, 66(1):7-30.

2. Siegel RL, Miller KD, Jemal A: Cancer statistics, 2020. CA: A Cancer Journal for Clinicians 2020, 70(1):7-30.

3. Weigelt B, Peterse JL, van 't Veer LJ: Breast cancer metastasis: markers and models. Nat Rev Cancer 2005, 5(8):591-602.

4. Friedl P, Wolf K: Tumour-cell invasion and migration: diversity and escape mechanisms. Nat Rev Cancer 2003, 3(5):362-374.

5. Kopp F, Hermawan A, Oak PS, Herrmann A, Wagner E, Roidl A: Salinomycin treatment reduces metastatic tumor burden by hampering cancer cell migration. Mol Cancer 2014, 13:16. 
6. Ni S, Hu J, Duan Y, Shi S, Li R, Wu H, Qu Y, Li Y: Down expression of LRP1B promotes cell migration via RhoA/Cdc42 pathway and actin cytoskeleton remodeling in renal cell cancer. Cancer Science 2013, 104(7):817-825.

7. Iyer MK, Niknafs YS, Malik R, Singhal U, Sahu A, Hosono Y, Barrette TR, Prensner JR, Evans JR, Zhao S: The landscape of long noncoding RNAs in the human transcriptome. Nature Genetics 2015, 47(3):199-208.

8. Ulitsky I, Bartel DP: lincRNAs: genomics, evolution, and mechanisms. Cel/ 2013, 154(1):26-46.

9. Schmitt AM, Chang HY: Long Noncoding RNAs in Cancer Pathways. Cancer Cel/ 2016, 29(4):452463.

10. Gupta RA, Shah N, Wang KC, Kim J, Horlings HM, Wong DJ, Tsai MC, Hung T, Argani P, Rinn JL et al: Long non-coding RNA HOTAIR reprograms chromatin state to promote cancer metastasis. Nature 2010, 464(7291):1071-1076.

11. Hu WL, Jin L, Xu A, Wang YF, Thorne RF, Zhang XD, Wu M: GUARDIN is a p53-responsive long noncoding RNA that is essential for genomic stability. Nat Cell Biol 2018, 20(4):492-502.

12. Massagué, Joan: TGF $\beta$ in Cancer. Cell 2008, 134(2):215-230.

13. Massague J, Seoane J, Wotton D: Smad transcription factors. Genes Dev 2005, 19(23):2783-2810.

14. Stolfi C, Marafini I, Simone VD, Pallone F, Monteleone G: The Dual Role of Smad7 in the Control of Cancer Growth and Metastasis. International Journal of Molecular Sciences 2013, 14(12):2377423790.

15. A N, M A, A M, T N, JL C, R H, S I, M K, NE H, CH H et al: Identification of Smad7, a TGFbeta-inducible antagonist of TGF-beta signalling. Nature 1997, 389(6651):631-635.

16. Ebisawa T, Fukuchi M, Murakami G, Chiba T, Tanaka K, Imamura T, Miyazono K: Smurf1 interacts with transforming growth factor-beta type I receptor through Smad7 and induces receptor degradation. Journal of Biological Chemistry 2001, 276(16):12477-12480.

17. Zhang S, Fei T, Zhang L, Zhang R, Chen F, Ning Y, Han Y, Feng XH, Meng A, Chen YG: Smad7 antagonizes transforming growth factor beta signaling in the nucleus by interfering with functional Smad-DNA complex formation. Molecular \& Cellular Biology 2007, 27(12):4488-4499.

18. Zhan MN, Yu XT, Tang J, Zhou CX, Wang CL, Yin QQ, Gong XF, He M, He JR, Chen GQ: MicroRNA-494 inhibits breast cancer progression by directly targeting PAK1. Cell Death \& Disease 2017, 8(1):e2529.

19. Minn AJ, Gupta GP, Siegel PM, Bos PD, Shu W, Giri D, Viale A, Olshen AB, Gerald WL, Massague J: Genes that mediate breast cancer metastasis to lung. Nature 2005, 436(7050):518-524.

20. Aragón E, Goerner N, Xi Q, Gomes T, Sheng G, Massagué J, Macias M: Structural Basis for the Versatile Interactions of Smad7 with Regulator WW Domains in TGF- $\beta$ Pathways. Structure 2012, 20(10):1726-1736.

21. Gronroos E, Hellman UHeldin CH, Ericsson J: Control of Smad7 Stability by Competition between Acetylation and Ubiquitination. Molecular Cel/ 2002, 10(3):483-493. 
22. Vardouli L, Moustakas A, Stournaras C: LIM-kinase 2 and Cofilin Phosphorylation Mediate Actin Cytoskeleton Reorganization Induced by Transforming Growth Factor- $\beta$. Journal of Biological Chemistry 2005, 280(12):11448-11457.

23. Oh JH, Yang JO, Hahn Y, Kim MR, Byun SS, Jeon YJ, Kim JM, Song KS, Noh SM, Kim S:

Transcriptome analysis of human gastric cancer. Mammalian Genome 2005, 16(12):942-954.

24. Liu B, Sun L, Liu Q, Gong C, Yao Y, Lv X, Lin L, Yao H, Su F, Li D: A Cytoplasmic NF-kB Interacting Long Noncoding RNA Blocks IKB Phosphorylation and Suppresses Breast Cancer Metastasis. Cancer Cell 2015, 27(3):370-381.

25. Tang J, Li Y, Sang Y, Bo Y, Lv D, Zhang W, Feng H: LncRNA PVT1 regulates triple-negative breast cancer through KLF5/beta-catenin signaling. Oncogene 2018.

26. Pardali K, Moustakas A: Actions of TGF-beta as tumor suppressor and pro-metastatic factor in human cancer. Biochim Biophys Acta 2007, 1775(1):21-62.

27. Seton-Rogers SE, Lu Y, Hines LM, Koundinya M, LaBaer J, Muthuswamy SK, Brugge JS: Cooperation of the ErbB2 receptor and transforming growth factor beta in induction of migration and invasion in mammary epithelial cells. Proc Natl Acad Sci U S A 2004, 101(5):1257-1262.

28. Shen X, Li J, Hu PP, Waddell D, Zhang J, Wang XF: The activity of guanine exchange factor NET1 is essential for transforming growth factor-beta-mediated stress fiber formation. Journal of Biological Chemistry 2001, 276(18):15362-15368.

29. Tsapara A, Luthert P, Greenwood J, Hill CS, Matter K, Balda MS: The RhoA Activator GEF-H1/Lfc Is a Transforming Growth Factor- $\beta$ Target Gene and Effector That Regulates a-Smooth Muscle Actin Expression and Cell Migration. Molecular Biology of the Cell 2010, 21(6):860-870.

30. Gregory PA, Bracken CP, Smith E, Bert AG, Wright JA, Roslan S, Morris M, Wyatt L, Farshid G, Lim YY: An autocrine TGF- $\beta / Z E B / m i R-200$ signaling network regulates establishment and maintenance of epithelial-mesenchymal transition. Molecular Biology of the Cell 2011, 22(10):1686-1698.

31. Bhola NE, Balko JM, Dugger TC, Kuba MG, Sánchez V, Sanders M, Stanford J, Cook RS, Arteaga CL: TGF- $\beta$ inhibition enhances chemotherapy action against triple-negative breast cancer. Journal of Clinical Investigation 2013, 123(3):1348-1358.

\section{Figures}


A

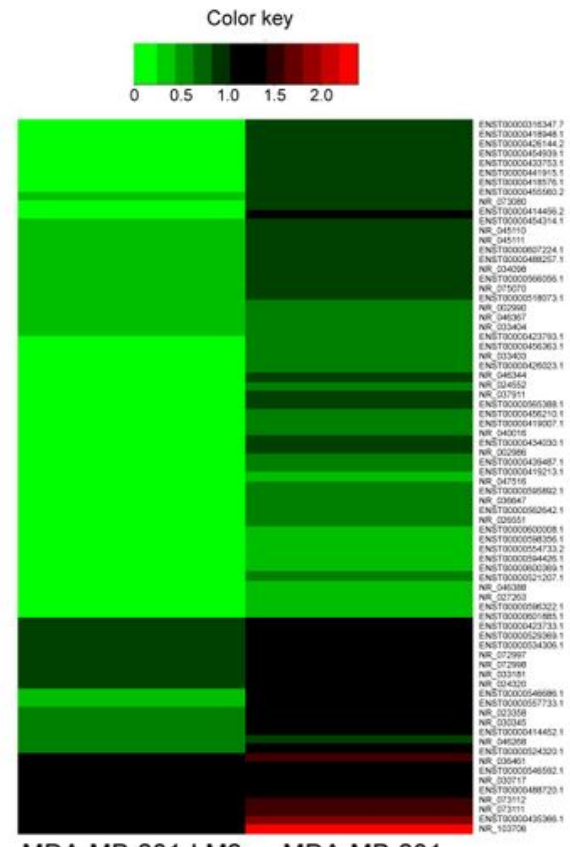

MDA-MB-231-LM2 MDA-MB-231
B

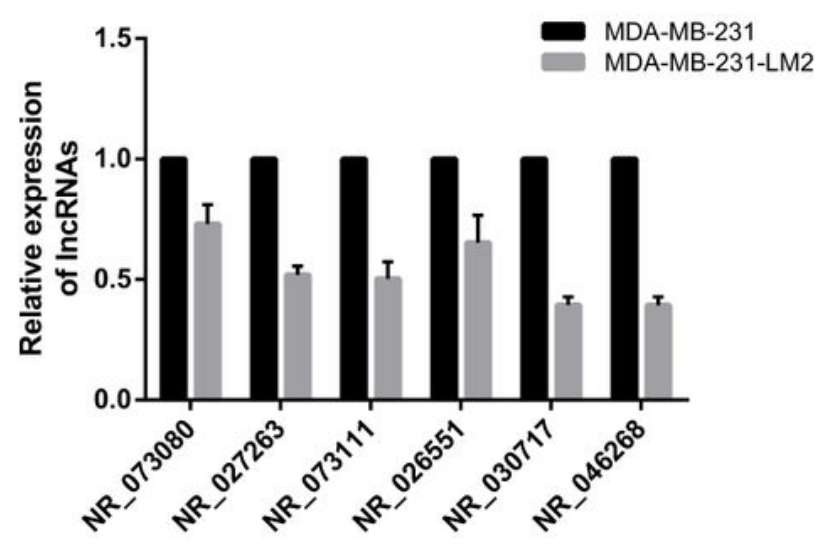

D

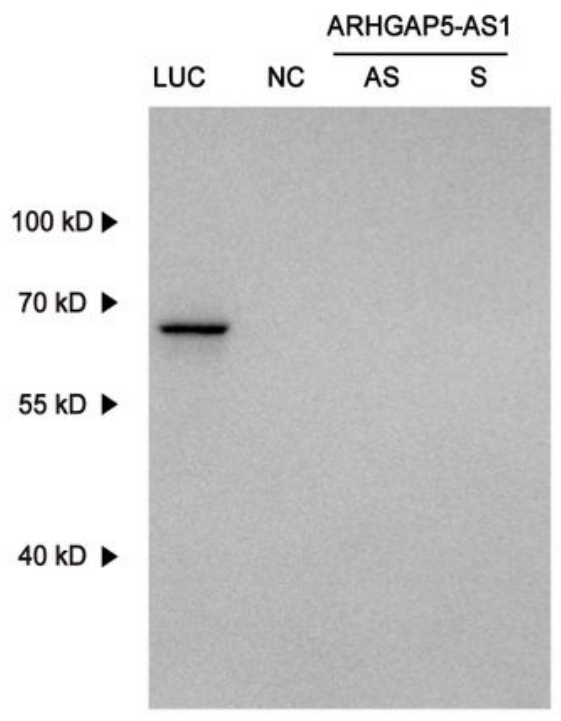

\section{Wang CL et al, Figure 1 亿}

\section{Figure 1}

LncRNA ARHGAP5-AS1 is downregulated in human breast cancer. (A) Heatmap of dysregulated RNA transcripts in MDA-MB-231 and MDA-MB-231-LM2 were shown. (B) The downregulation level of six LncRNA candidates in MDA-MB-231-LM2 cells was dected by qRT-PCR, compared to MDA-MB-231 cells. (C) The effects of NR_027263, NR_046268, NR_030717 on the migration ability of MDA-MB-231 cells 
were detected by transwell migration assays. (D) in vitro translation of plasmids containing luciferase, negative control, antisense and sense ARHGAP5-AS1 were detected by western blot.

A
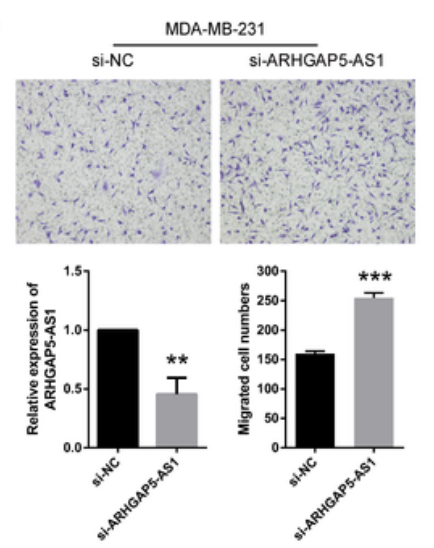

B
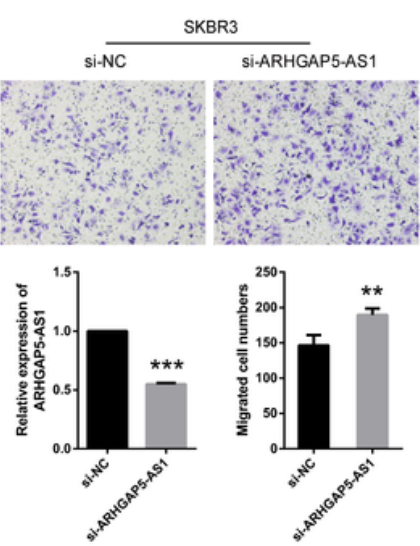

C
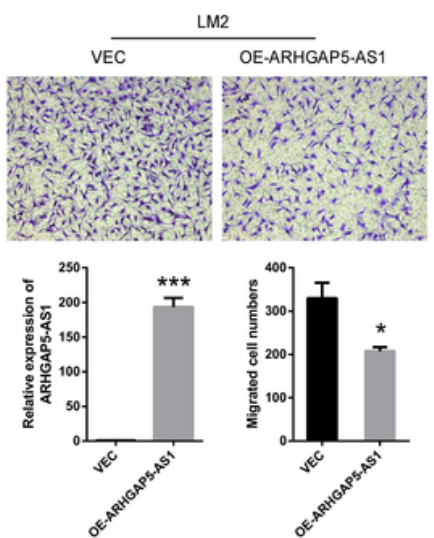

D
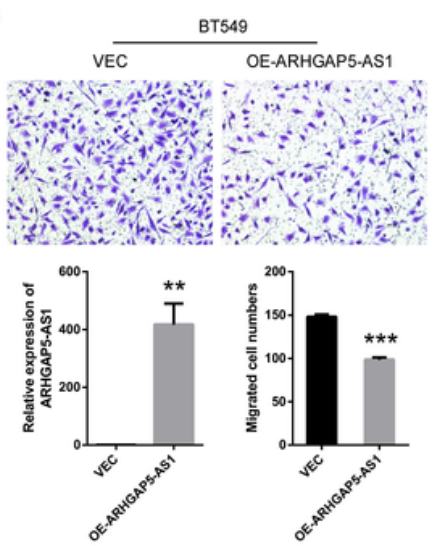

E
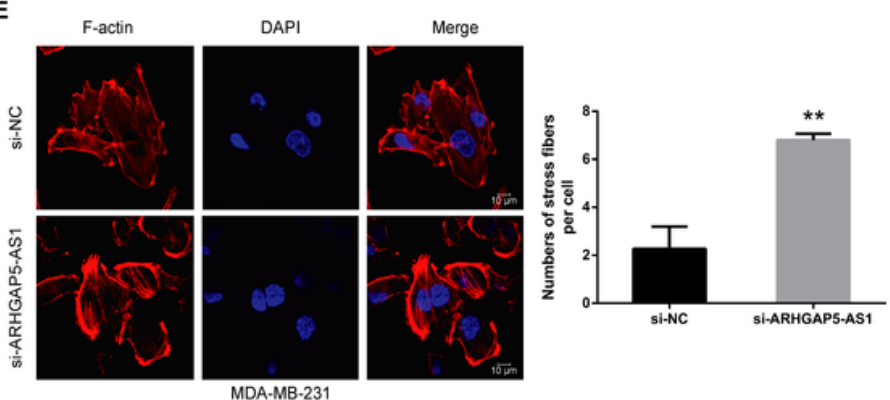

G
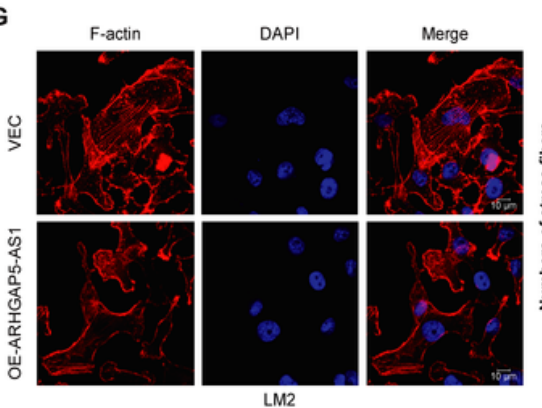

$\mathbf{F}$
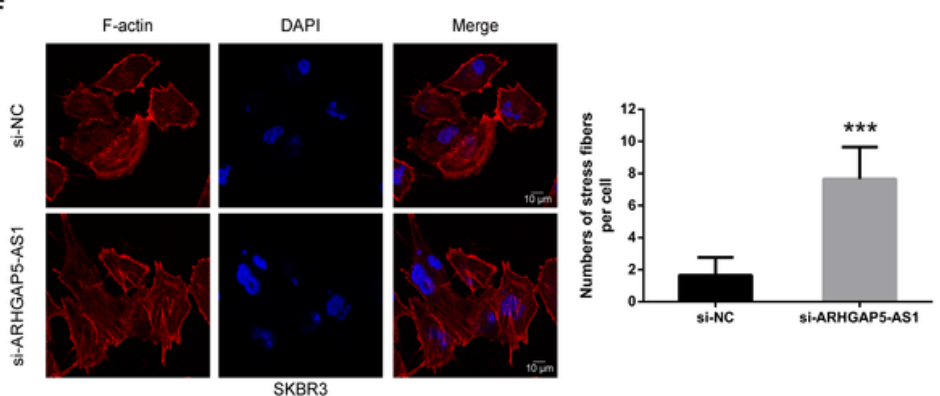

H
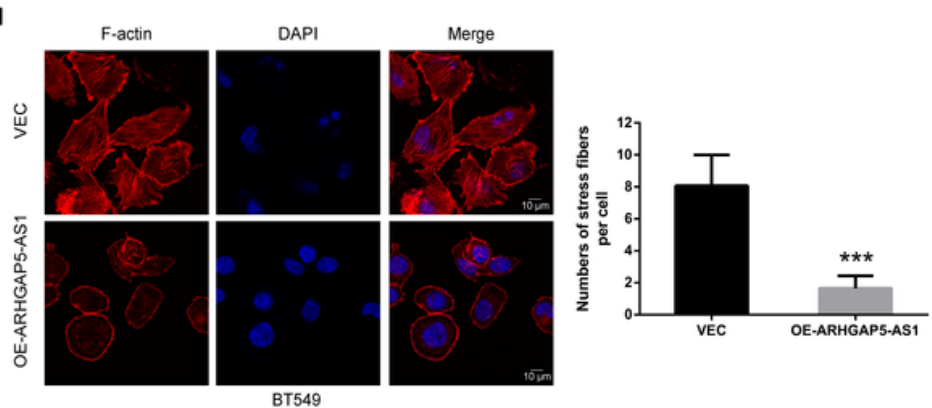

Wang CL et al, Figure 2 介

Figure 2

ARHGAP5-AS1 inhibits migration of breast cancer cells. Transwell migration assays of knockdown of ARHGAP5-AS1 in (A) MDA-MB-231 cells and (B) SKBR3 cells as well as over-expression of ARHGAP5-AS1 in (C) LM2 cells and (D) BT549 cells were measured and the results were expressed as the number of migrated cells per field. Knockdown \& over-expression efficiencies of ARHGAP5-AS1 in each cell line were shown below. Immunofluorescence of F-actin of knockdown of ARHGAP5-AS1 in (E) MDA-MB-231 cells and (D) SKBR3 cells as well as over-expression of ARHGAP5-AS1 in (F) LM2 cells and (G) BT549 cells were performed and the results were presented as the number of stress fibers per cell. The error bars in all graphs represented SD and each experiment was repeated three times independently. ${ }^{\star} \mathrm{p} \otimes 0.05,{ }^{* \star} \mathrm{p} \otimes 0.01$, $\star \star \star \mathrm{p} \otimes 0.001$. 
A

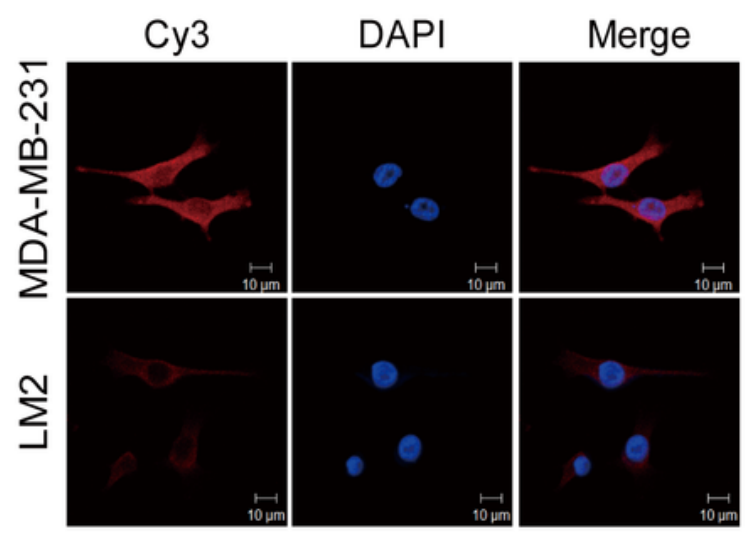

C

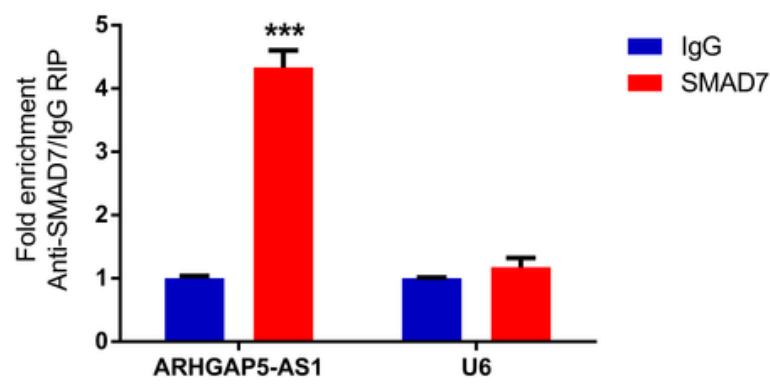

$\begin{array}{lll}\text { Input } \quad \lg \text { S } & \text { SMAD7 } \\ \text { Anti-SMAD7-GAPDH } & \\ & \end{array}$

D

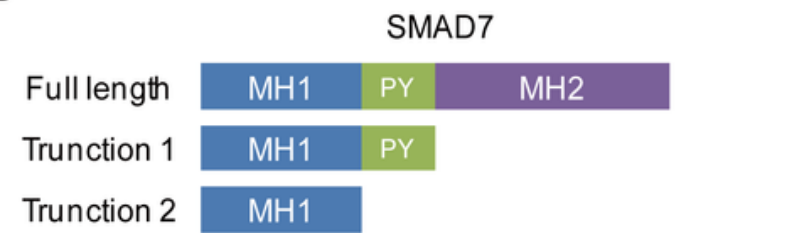

B

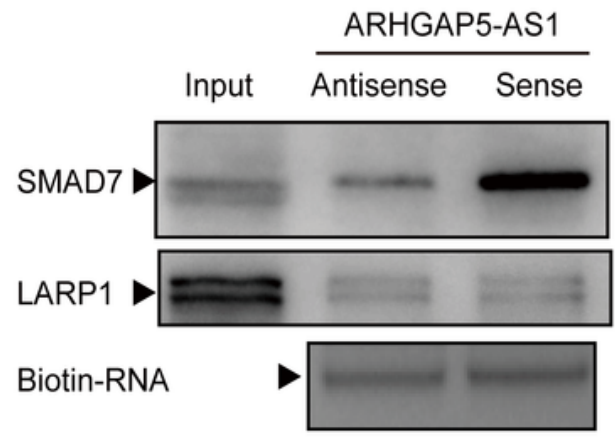

E
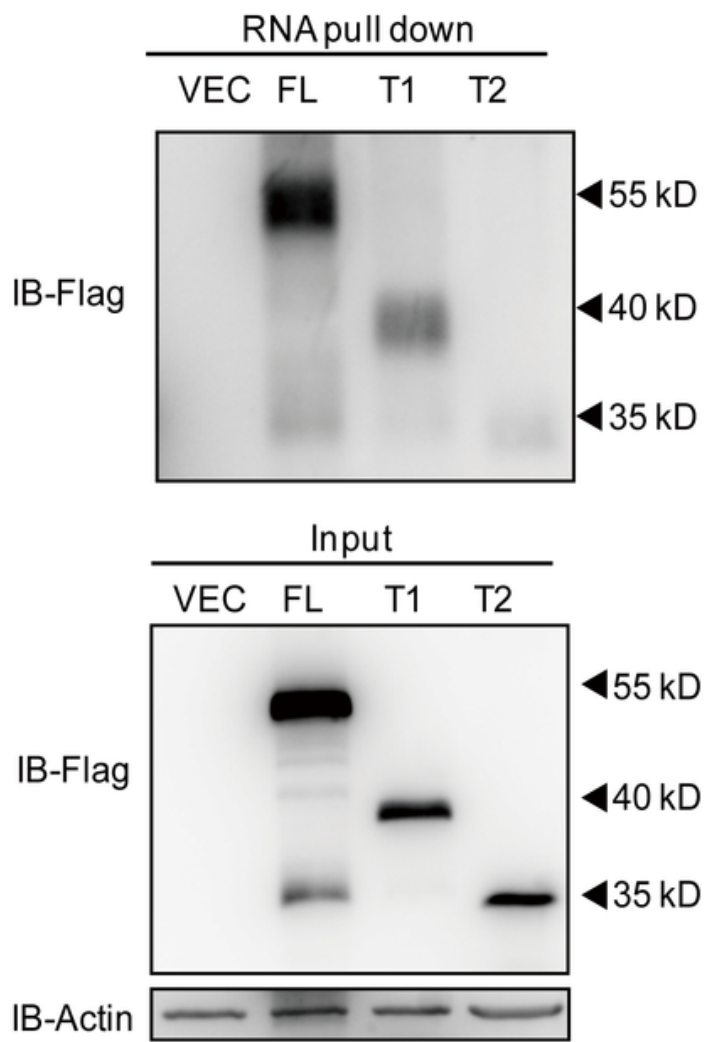

Wang CL et al, Figure 3 介

\section{Figure 3}

ARHGAP5-AS1 interacts with the PY motif of SMAD7. (A) The subcellular location of ARHGAP5-AS1 in MDA-MB-231 (upper) and LM2 (lower) cells were detected by RNA FISH with Cy3 labeled probes. (B) Identification of ARHGAP5-AS1 interacting proteins by RNA pull down assay was detected by western blot. (C) RNA immunoprecipitation assay was performed using SMAD7 and IgG antibody in MDA-MB-231 cells. Pulled-down ARHGAP5-AS1 and U6 were detected by qRT-PCR. (D) The graph showed structures of 
full-length SMAD7 and truncation 1 \& 2. (E) RNA pull down assay was performed to detect the interaction between ARHGAP5-AS1 and flag-tagged SMAD7, truncation 1 \& 2.

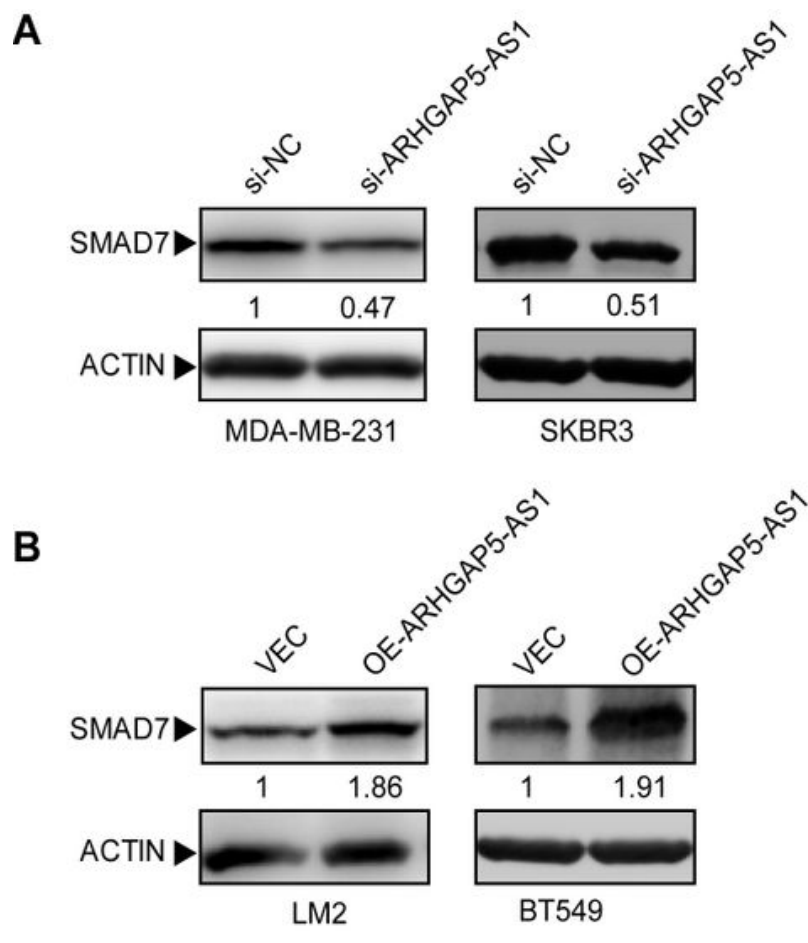

C

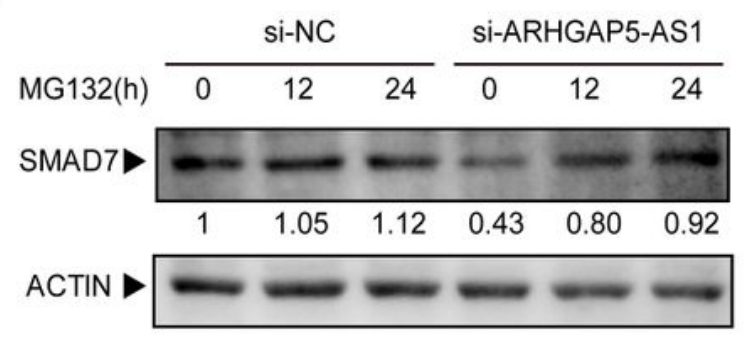

D

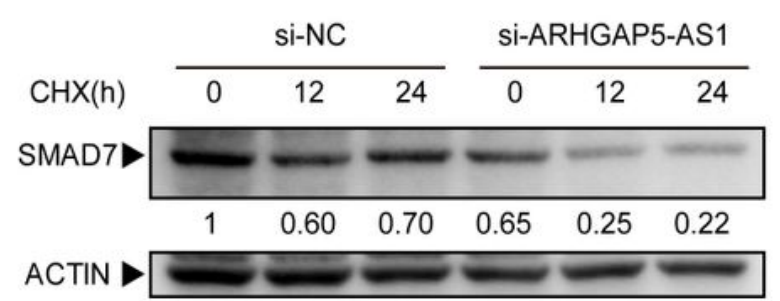

E
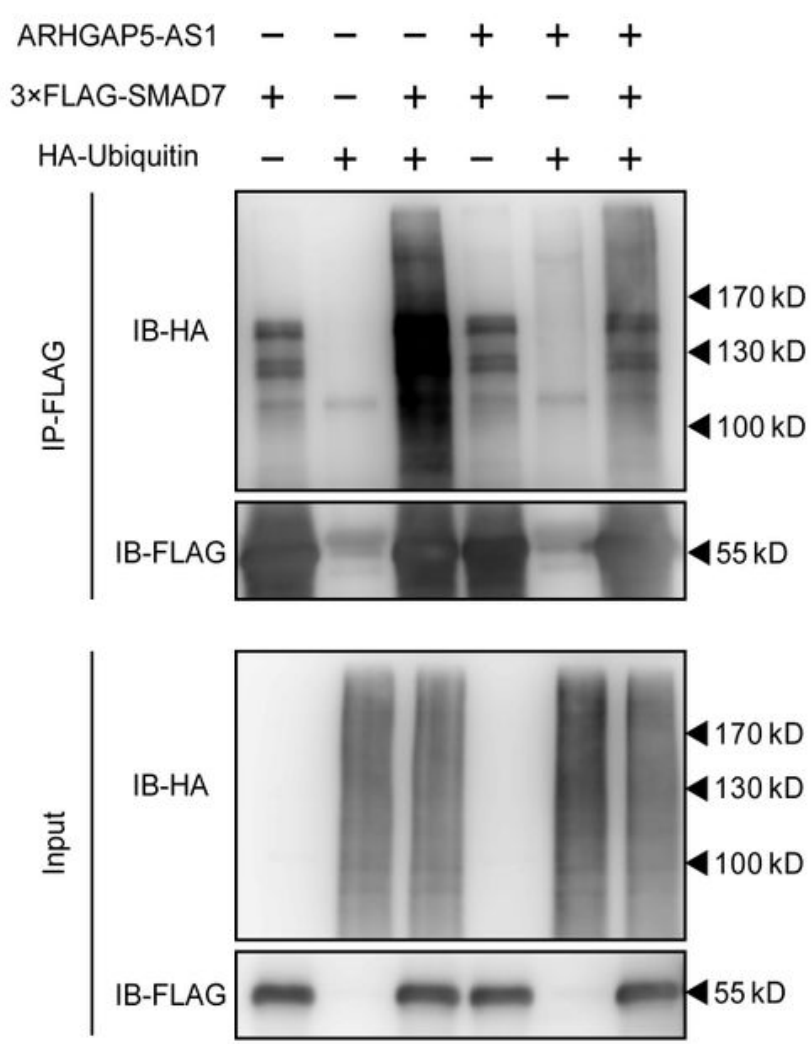

$\mathbf{F}$
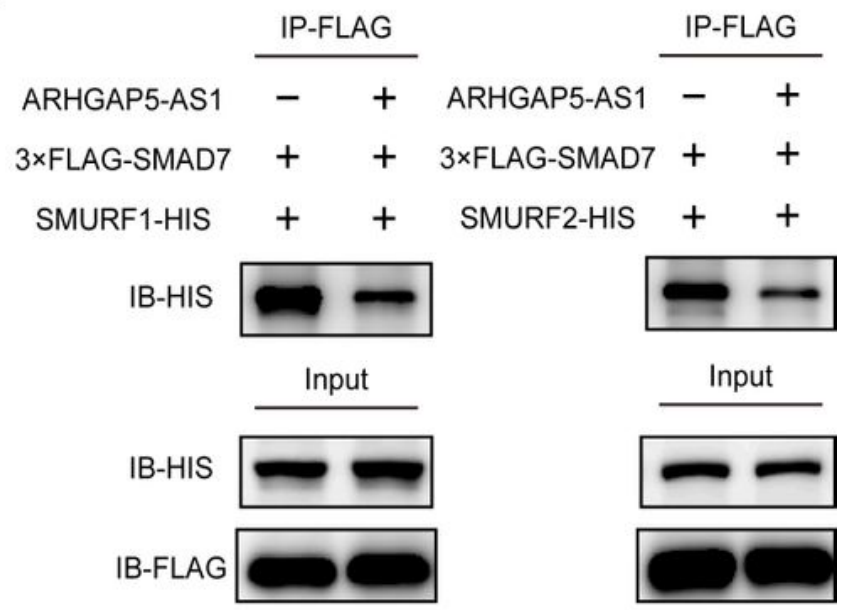

\section{Wang CL et al, Figure 4 亿}

\section{Figure 4}

ARHGAP5-AS1 stabilizes SMAD7 via inhibition of ubiquitination of SMAD7. (A) The protein level of SMAD7 was detected by western blot in MDA-MB-231 cells and SKBR3 cells with knockdown of ARHGAP5-AS1. (B) The protein level of SMAD7 was detected by western blot in LM2 cells and BT549 
cells with over-expression of ARHGAP5-AS1. (C) Knockdown of ARHGAP5-AS1 in MDA-MB-231 cells with treatment of $20 \mu \mathrm{M}$ MG132. (D) Knockdown of ARHGAP5-AS1 in MDA-MB-231 cells with treatment of 40 $\mu \mathrm{M}$ CHX. (E) Immunoprecipitation was performed to enrich ubiquitin-SMAD7 complex in 293T cells with indicated transfections. (F) Immunoprecipitation of FLAG was performed to enrich FLAG-tagged SMAD7 and HIS-tagged SMURF1/SMURF2 complex with or without over-expression of ARHGAP5-AS1 in 293T cells.

A

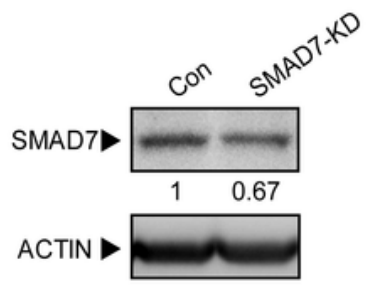

C
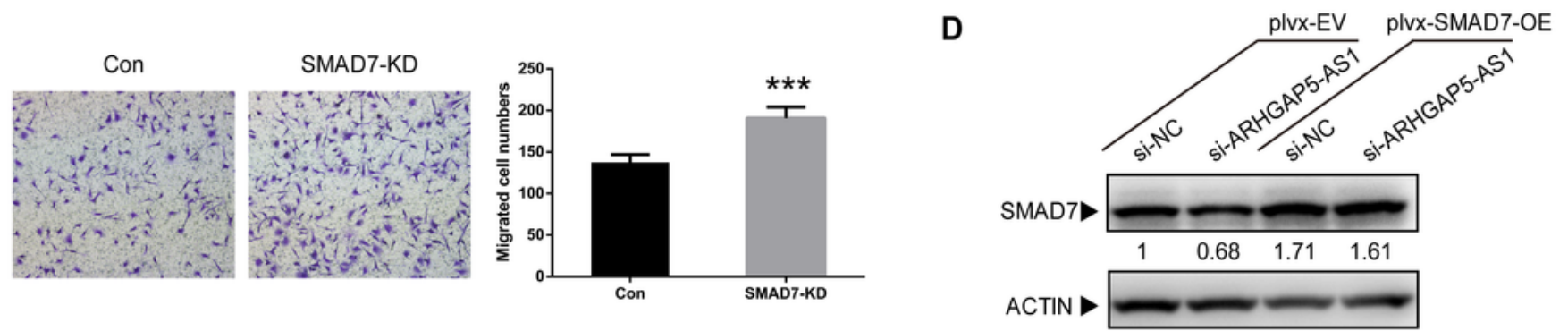

B
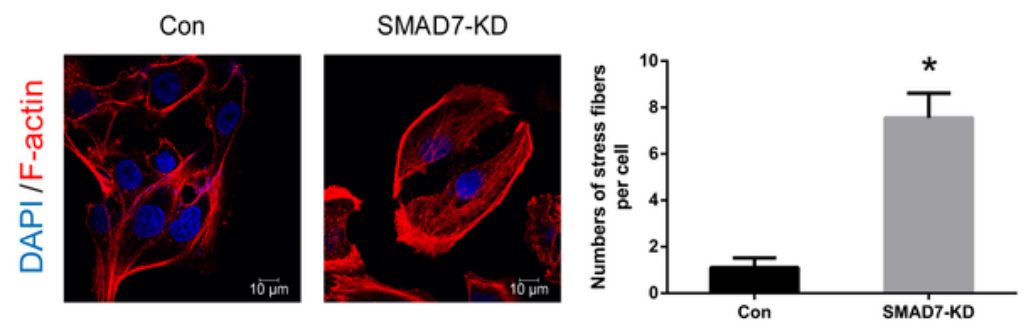

E

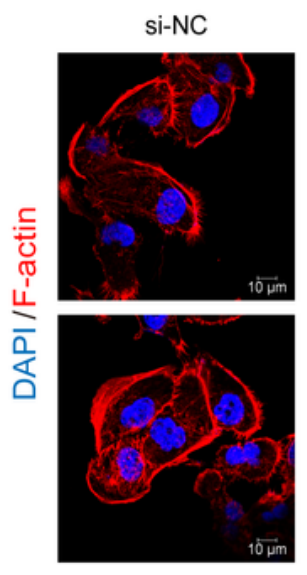

si-ARHGAP5-AS1
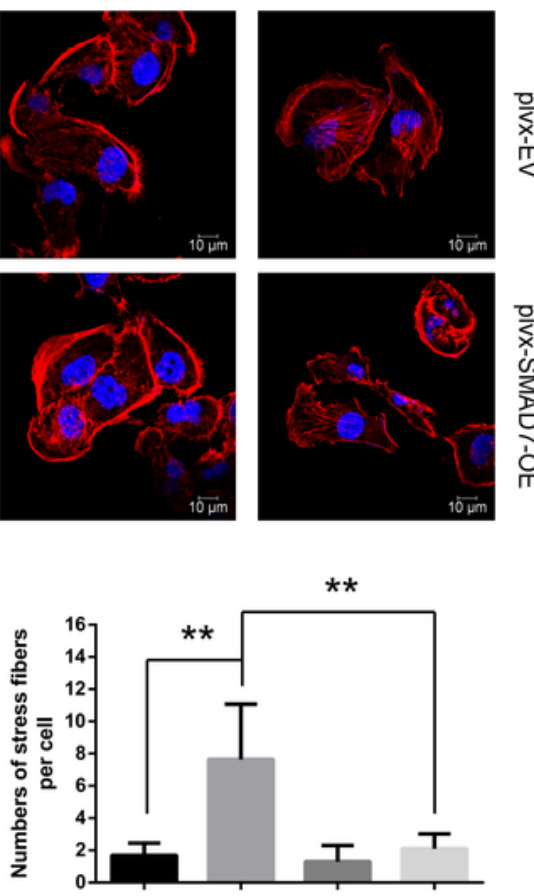

si-ARHGAP5-AS1

plvx-SMAD7-OE
F
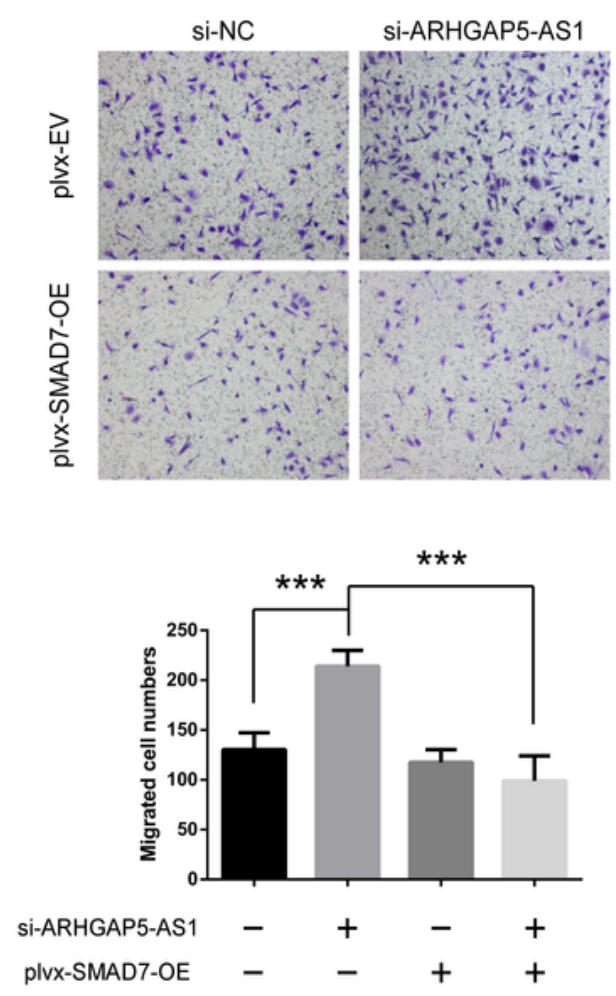

Wang CL et al, Figure 5 亿 
SMAD7 mediates the negative effect of ARHGAP5-AS1 on cell migration. (A) The knockdown efficiency of SMAD7 DNA antisense oligo was detected by western blot. (B) Immunofluorescence of F-actin of SMAD7 knockdown in MDA-MB-231 cells was performed and the results were presented as the number of stress fibers per cell. (C) Transwell migration assay of SMAD7 knockdown was measured and the results were expressed as the number of migrated cells per field. (D) The protein level of SMAD7 was detected by western blot in plvx-EV and plvx-SMAD7-OE cells with si-NC or si-ARHGAP5-AS1 transfection. (E) Immunofluorescence of F-actin was performed with indicated transfections. (F) Transwell migration assay was performed with indicated transfections. The error bars in all graphs represented SD and each experiment was repeated three times independently. ${ }^{\star} p \otimes 0.05, \star \star p \vee 0.01, \star \star \star p \otimes 0.001$ 
A

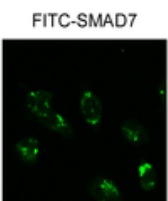

DAPI
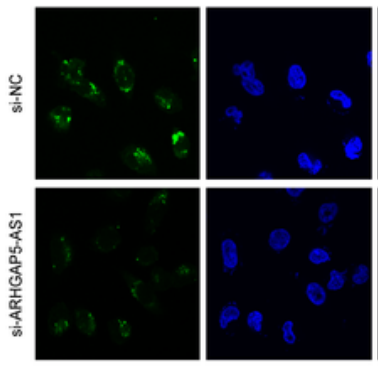
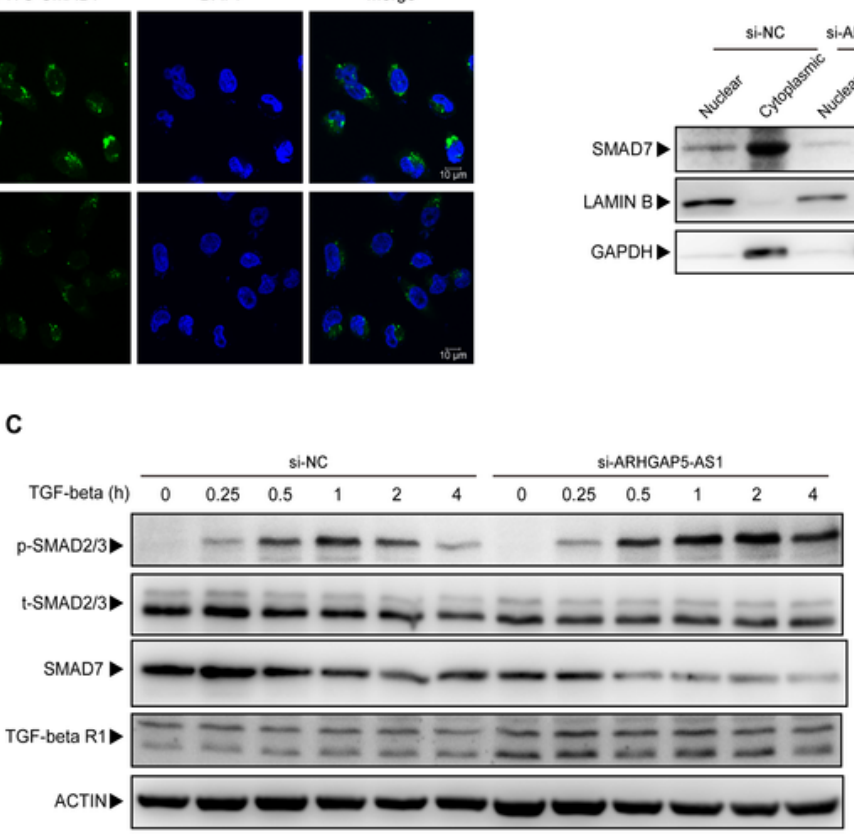

D

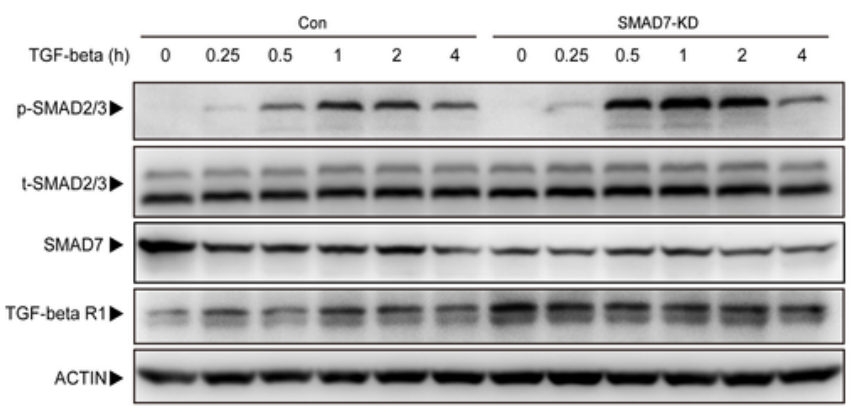

E

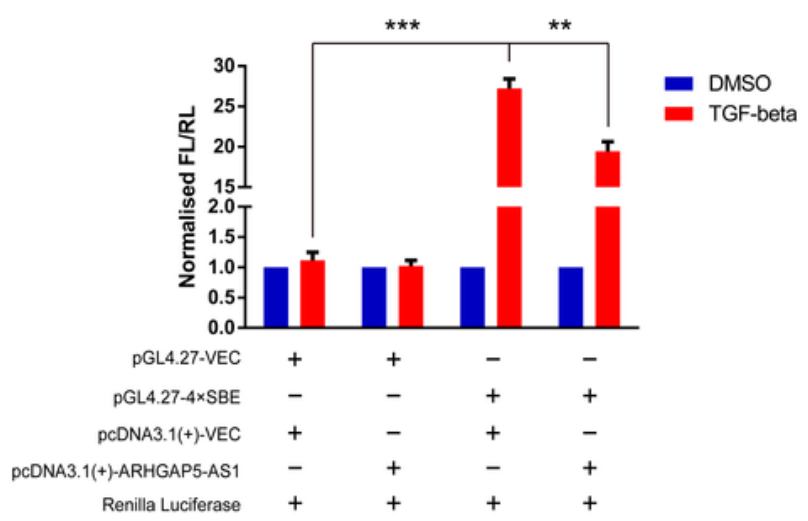

Wang CL et al, Figure 6 介

\section{Figure 6}

Knockdown of ARHGAP5-AS1 promotes TGF- $\beta$ signaling via SMAD7. (A) Immunofluorescence of SMAD7 with FITC-conjugated antibody was performed to detect location of SMAD7 in MDA-MB-231 cells. (B) Nuclear and cytoplasmic SMAD7 was detected by western blot, LAMIN B and GAPDH were used as loading controls. (C) Effects of ARHGAP5-AS1 knockdown on the activation of TGF- $\beta$ signaling were detected by western blot. (D) Effects of SMAD7 knockdown on the activation of TGF- $\beta$ signaling were 
detected by western blot. (E) Luciferase reporter assays were performed in 293T cells with indicated transfections. The error bars in all graphs represented SD and each experiment was repeated three times independently. ${ }^{*} \otimes \otimes 0.05, * * p \otimes 0.01, * \star \star p \otimes 0.001$

\section{Supplementary Files}

This is a list of supplementary files associated with this preprint. Click to download.

- Authorlnfomation.docx

- LegendsforSupplementalFigures.docx

- Figures2.tif

- Figures1.tif 